\title{
Trilogie de la personne. La pensée philosophique et l'engagement politique de Denis de Rougemont
}

\section{Damiano Bondi}

Traducteur : Elisa Grimaldi

\section{OpenEdition \\ Journals}

Édition électronique

URL : https://journals.openedition.org/edl/1889

DOI : $10.4000 /$ edl. 1889

ISSN : 2296-5084

\section{Éditeur}

Université de Lausanne

\section{Édition imprimée}

Date de publication : 15 décembre 2019

Pagination : 163-180

ISBN : 978-2-940331-72-7

ISSN : 0014-2026

\section{Référence électronique}

Damiano Bondi, «Trilogie de la personne. La pensée philosophique et l'engagement politique de Denis de Rougemont », Études de lettres [En ligne], 311 | 2019, mis en ligne le 15 décembre 2021, consulté le 16 décembre 2021. URL : http://journals.openedition.org/edl/1889 ; DOI : https://doi.org/10.4000/edl. 1889

Ce document a été généré automatiquement le 16 décembre 2021.

(c) Études de lettres 


\title{
Trilogie de la personne. La pensée philosophique et l'engagement politique de Denis de Rougemont
}

\author{
Damiano Bondi \\ Traduction : Elisa Grimaldi
}

1 La première tâche des intellectuels est, aujourd'hui, de conduire une critique des mythes collectivistes nés de la maladie de la personne. Puis il s'agit de retrouver une définition concrète de la personne. Enfin de la traduire en institutions et coutumes. $\mathrm{Ou}$, tout au moins, d'indiquer les limites, la formule et les buts de ces institutions ${ }^{1}$.

Dans cette déclaration, nous pouvons voir une synthèse de toute la vie intellectuelle de Denis de Rougemont, ainsi que de son travail philosophique et de son engagement politique. En effet, tout au long de sa production (tant théorique que théologique, littéraire que sociologique), il est possible d'identifier trois pôles thématiques qui se recoupent tout en se distinguant, qui structurent et qui déterminent leur développement, tant au sens diachronique que synchronique. Le premier noyau thématique, celui de la «critique des mythes collectivistes nés de la maladie de la personne ", remonte notamment à la première phase de sa pensée, qui va des Méfaits de l'instruction publique (1929) à La part du diable (1942), en passant par Politique de la personne (1934), Penser avec les mains (1936) et L'amour et l'Occident (1939). Le deuxième, qui consiste à "trouver une définition concrète de la personne", se trouve plus facilement dans ses œuvres des années quarante et cinquante, notamment Les personnes du drame (1944) et L'aventure occidentale de l'homme (1957). Enfin, le troisième, qui vise à «traduire » la conception de la personne en « institutions ou coutumes » en indiquant " les limites, la formule et les objectifs », est propre surtout aux dernières années de la production du philosophe, à partir des années cinquante, avec des œuvres telles que L'un et le divers ou la cité européenne (1970) et L'avenir est notre affaire (1977) ${ }^{2}$. Nous explorerons donc le contenu de ces œuvres, afin de définir l'architecture générale d'une conception philosophico-politique des plus riches et des plus articulées du $\mathrm{XX}^{\mathrm{e}}$ siècle. 


\section{La personne contre les mythes collectifs}

La perspicacité philosophique de Rougemont s'exprime dans les premières années sous la forme d'un esprit critique, et son premier objectif polémique est l'institution scolaire. En fait, marqué négativement par son expérience personnelle à l'école de Couvet dans le canton de Neuchâtel, il a ressenti le besoin, à l'âge de vingt-deux ans, d'en condamner radicalement les principes de base et, avec eux, la même conception moderne de l'enseignement public. Sorti en 1929, Les méfaits de l'instruction publique est un pamphlet violent "au rythme du plaidoyer et au timbre de l'invective $»^{3}$. On n'y trouve aucune trace de ce style sobre, profondément analytique et méticuleux qui caractérisera le Rougemont de L'amour et l'occident. Ici, il n'y a que l'« écrit d'un jeune homme en colère $"^{4}$, comme il l'a lui-même reconnu des années plus tard dans son commentaire de l'œuvre ${ }^{5}$. Mais nous percevons aussi un homme capable, comme peu d'autres, d'aller droit au cœur des problèmes et d'indiquer une solution possible. De plus, il n'est pas exagéré d'affirmer que dans cet écrit de jeunesse, nous pouvons déjà trouver en germe les principes fondamentaux qui deviendront plus tard, correctement repensés et reformulés, les pierres angulaires de sa pensée, en particulier la personne.

Quand Rougemont déclare que «l'école, sous sa forme actuelle, remplit suffisamment son rôle politique et social, qui est de fabriquer des électeurs", limitant ainsi "l'homme au citoyen", ce qui imposerait de "dépasser le citoyen, de retrouver l'homme tout entier $»^{6}$, il est impossible de ne pas voir dans cet « homme entier» un embryon de ce qui deviendra ensuite pour lui la «personne humaine authentique ».

5 Certes, afin que Rougemont parvienne à une conception mûre de la personne, les rencontres à Paris d'Alexandre Marc, de Robert Aron et plus tard d'Emmanuel Mounier seront nécessaires; mais il serait faux de diminuer sa contribution spécifique à l'établissement du premier noyau des personnalistes français. Par exemple, la célèbre expression «désordre établi» (qui indiquerait polémiquement l'égalitarisme démocratique et l'individualisme bourgeois), généralement attribuée à Mounier, se retrouve déjà dans Les méfaits de l'instruction publique et est donc inventée par Rougemont un an avant son déménagement à Paris.

6 En outre, il est raisonnable d'affirmer que l'accent mis par les auteurs personnalistes sur le concept de "vocation", en tant que dimension constitutive de l'expérience existentielle et religieuse de la personne, constitue davantage un héritage du protestant Rougemont que du catholique Mounier. C'est en effet le premier qui, en 1934, dans Esprit, écrit de manière péremptoire : «La personne est une vocation " ${ }^{7}$. Autrement dit, selon l'auteur, chaque personne a une vocation particulière et unique, différente de toutes les autres :

La personne est le témoignage d'une vocation reçue et obéie. Je suis personne dans la mesure où mon action relève de ma vocation, fût-ce au prix de la vie de mon individu ${ }^{8}$.

7 Rougemont établit ici une relation dialectique entre personne et individu qui sera aussi celle de Mounier. Ce dernier écrira en effet que l'individu reste le soutien concret qui est nécessaire au développement de la personne, " par où lui viennent ses nourritures obscures $"^{9}$; Rougemont le rejoindra et il précisera que : 
[...] la personne est distincte de l'individu, mais ne peut pas exister sans lui.

L'individu n'est pas le contraire de la personne, c'est plutôt la condition

nécessaire ${ }^{10}$.

8 Il s'agirait alors de faire en sorte que "la personne unique s'édifie dans l'individu " ${ }^{11}$, c'est-à-dire que « la personne est en nous l'être spirituel, responsable d'une vocation, et trouvant là son unité en dépit des contradictions dont peut souffrir l'individu, c'est-àdire l'être naturel $»^{12}$.

Récemment, le philosophe et théologien Robert Spaemann a déclaré, d'une manière tout à fait analogue, que si les animaux sont naturels, les hommes ont une nature, c'està-dire qu'ils se rapportent à leur "datité " (par rapport à leur individu) selon les modalités de l'avoir et du disposer de ${ }^{13}$. Cependant, cela ne signifie pas du tout que la nature individuelle doit être exclue puisqu'elle est la condition nécessaire au développement de la personne; elle est au contraire appelée à s'y incarner ou à disposer de son individu selon sa vocation. Il est donc nécessaire que chaque homme se confronte avec ses propres limites concrètes, avec ses propres contraintes physiques, afin de parvenir à une liberté qui ne prend pas la forme d'un volontarisme abstrait de l'esprit, mais plutôt d'une action positive et efficace sur une réalité spécifique. «Je nommerai libre un homme qui se possède $»^{14}$, dit fermement Rougemont.

Cependant, c'est précisément cet appel à la liberté personnelle qui fait peur à l'homme. Ainsi, il l'évite souvent, en renonçant à son essence la plus profonde et en se réfugiant en même temps sous la protection de "léviathans", afin qu'ils le libèrent de cet écrasant sens des responsabilités à l'égard de sa propre vie. C'est l'interprétation rougemontienne des totalitarismes contemporains $d u X X^{e}$ siècle. Ce ne sont que des «mythes» forgés par la peur des hommes de devenir des personnes authentiques, de répondre à leur vocation, d'accepter le risque d'une existence libre.

11 Staline et Hitler, dans cette perspective, incarnent un modèle de société fondé sur l'aliénation de la personne. Le paradigme sous-jacent commun est représenté par l'idéologie du collectivisme, selon laquelle :

Le bien commun passe avant le bien particulier [...] et l'homme ne vaut que par son activité au service du peuple ou du prolétariat, c'est-à-dire, concrètement, au service du Parti, qui selon la parole du Führer est «la volonté organisée de la nation $»^{15}$.

12 C'est la nation qui a un destin, pas l'homme qui fait l'histoire; c'est l'État qui doit être servi par le citoyen et non le citoyen qui bénéficie des services que l'État lui offre ; c'est l'État-nation (un autre terme inventé par les personnalistes) qui fournit un horizon de sens aux actions humaines, et non plus un appel transcendant à tout ordre humain. Donc, en dernière analyse, «les mythes collectifs n'expriment rien de plus qu'une certaine attitude, l'attitude démissionnaire de l'homme en fuite devant sa vocation ${ }^{16}$.

Or, il n'y a pas que les mythes politiques qui sont les plus faciles à démasquer, précisément parce qu'ils prennent une forme extérieure et visible. Il existe des démissions spirituelles bien plus profondes, ancrées dans la culture occidentale, comme le grand mythe de l'amour-passion, auquel Rougemont consacre son plus célèbre volume, L'amour et l'Occident (1939). D'ailleurs, on trouve dans cet ouvrage une conception du «mythe » beaucoup plus réfléchie et consciente, qui subit l'influence de l'entourage de Rougemont au Collège de sociologie ${ }^{17}$. En effet, c'est Rougemont qui admet que, bien que de profondes différences le séparent de la pensée de Bataille, il n'avait pas envisagé, avant d'avoir lui-même fréquenté le Collège, le thème de l'amour 
autrement que comme une affaire privée et individuelle, en omettant les traits collectifs et mythiques-sacraux ${ }^{18}$. Des traits qui, au contraire, correctement mis en évidence et argumentés avec expertise, contribueront à faire de L'amour et l'Occident l'une des plus grandes éditions à succès de l'époque, en termes de vente, de diffusion et d'influence culturelle ${ }^{19}$. La thèse centrale du volume est révolutionnaire : la conception occidentale de l'amour n'est rien d'autre qu'une forme séculaire de doctrines spiritualistes gnostiques et manichéennes qui, en raison de l'influence du catharisme sur la poésie courtoise, se sont ensuite répandues dans notre littérature. Par conséquent, ce qui semblerait à première vue être l'exaltation de la passion charnelle ne serait en réalité qu'un masque de l'Éros perpétuellement inassouvi, du désir qui doit être toujours entravé pour qu'il continue de brûler. Et le résultat nécessaire de ce « désir de désirer » sera la mort corporelle, car ce n'est qu'« en esprit » qu'il sera possible de réaliser cette fusion d'amants entravée en premier lieu par l'emprisonnement de la finitude corporelle.

Eros et Thanatos seraient donc étroitement liés, car le désir d'amour dissimulerait un désir de mort bien plus profond. Cependant, ce dernier resterait quelque chose d'inavouable pour la conscience des amoureux. Il serait donc nécessaire d'élaborer des mythes visant d'une part à exprimer et à transmettre le contenu doctrinal de la passion mortelle et, d'autre part, à en contenir le potentiel destructeur par le biais de formes socialement acceptables. Le "mythe» de Tristan et Iseut est interprété dans cette perspective; il est considéré par Rougemont comme l'«archétype » de l'amourpassion: un amour qui semble aspirer à sa propre réalisation, alors qu'il l'évite toujours, parce qu'il vit de l'obstacle, et non de la présence concrète de l'autre. L'épée que Tristan met entre son corps et celui d'Iseut, tous deux nus dans la forêt, témoigne de cette recherche de quelque chose qui sépare les deux amants. De même, le mariage de Tristan avec l'autre Iseut, celle " aux mains blanches », ne semble s'expliquer que si on reconnaît là, avec Rougemont, la volonté des amants de rester toujours à distance afin de pouvoir se désirer pour l'éternité, en même temps que se trouve dénoncée la fidélité conjugale comme tombeau de l'Éros céleste.

En fin de compte, pour Rougemont, "Tristan n'aime pas Iseut mais l'amour même, et au-delà de cet amour, la mort, c'est-à-dire la seule délivrance du moi coupable et asservi $»^{20}$. Mais l'amour pour la mort, nous l'avons dit, est inavouable et doit donc trouver un moyen symbolique de s'exprimer. Dans le mythe de Tristan, cette fonction est remplie par le filtre magique, qui est la cause apparente du désir d'amour. Ce filtre revêt la passion de fatalité, ce qui en fait une condamnation contre laquelle les amoureux ne peuvent pas se rebeller. Mieux encore, le filtre est "l'alibi de la passion $»^{21}$ : un dieu sans nom, une force aveugle qui permet aux amoureux de ne pas se sentir responsables de leurs tourments.

Ils s'aiment, mais ils ne s'aiment point; ils ont péché, mais ils ne peuvent s'en repentir, puisqu'ils ne sont pas responsables ; ils se confessent, mais ne veulent pas guérir, ni même implorer leur pardon...22

L'amour-passion serait donc un grand «mythe collectif » de l'Occident, profondément dépersonnalisant. Dans l'amour-passion, l'autre n'est pas aimé tel qu'il est, dans la réalité irréductible de son espoir et de son angoisse, mais comme prétexte à une glorification narcissique aboutissant à la mort. Au contraire, l'amour du prochain, en lequel consiste l'agapè chrétienne, suppose «l'acceptation du différent, et donc de 
l'incomplet, la prise sur le concret dans ses limitations $»^{23}$; il est ainsi capable de fonder la personne en préservant son caractère unique et sa diversité.

Ici donc, le personnalisme rougemontien apparaît comme une attitude profondément réaliste pour faire face à la différence et à la diversité, alors que les tentatives idéalistes d'atteindre un bien commun, égal pour tous, sont nécessairement vouées à l'échec, aboutissant à des totalitarismes politiques ou à une massification homogénéisante.

\section{Une définition concrète}

Comment est-il possible de donner une définition concrète ? Une définition est quelque chose de conceptuel, d'immatériel. Rougemont affirme pourtant la nécessité de parvenir à une « définition concrète de la personne ». À bien des égards, d'après lui, on ne peut donner une définition concrète que de la personne, cette dernière étant une réalité essentiellement dialectique, en tension perpétuelle entre sa propre condition naturelle/individuelle et sa propre réalisation vocationnelle :

L'apparition de la personne est liée à l'apparition d'une tension. Car d'une part elle est déterminée par les conditions données, d'autre part elle a pour but de les dépasser et de les rendre créatrices ${ }^{24}$.

Dans cette conception, il est possible de reconnaître des influences fortement kierkegaardiennes, qui sont par ailleurs ouvertement déclarées. Le penseur danois, à qui Rougemont, dans Les personnes du drame, consacre certaines de ses pages les plus denses et les plus originales ${ }^{25}$, est présenté comme le seul capable de lutter contre l'Aufhebung hégélien, en proposant comme clé herméneutique fondamentale de l'existence une " dialectique [...] simultanée, irréductible, vivante ${ }^{26}$, où c'est la même tension entre les deux pôles de l'opposition qui nourrit la vitalité créatrice qui en découle, et non leur résolution en une synthèse pacifique : tertium non datur.

Or le modèle d'une telle conception de la personne est fourni par le dogme chrétien de l'Incarnation qui " porte à l'extrême la coexistence des contraires, dans l'impensable définition de la Personne de Jésus-Christ comme "vrai Dieu et vrai homme "à la fois, selon les formules conciliaires de Nicée et de Chalcédoine ${ }^{27}$. L'Incarnation est véritablement, pourrait-on dire, une définition concrète de la personne, puisque dans le Christ, on mesure la possibilité d'assumer en soi le drame crucial de l'existence, ne faisant de cette manière qu'un instrument de salut et de réalisation authentiques. Cette idée est une sorte de constante de la pensée rougemontienne. Il la présente déjà clairement dans son article paru dans Esprit, intitulé précisément «Définition de la personne » :

L'imagination de la personne à l'état pur resterait à nos yeux une espèce d'utopie ontologique, si la Révélation n'en attestait l'acte historique. L'incarnation totale de Dieu dans l'Homme, l'humanité parfaite de Jésus-Christ est la limite atteinte de la personne dans l'histoire, le fait extrême, le concretissimum à partir duquel nous puissions penser activement la personne, c'est-à-dire réduire la distance qui sépare notre vie de notre vocation ${ }^{28}$.

21 Penser activement la personne signifie donc en faire, selon une terminologie reprise par Éric Weil, «le foyer d'une attitude». Paul-Louis Landsberg la désignerait par le terme d'Ausweis, c'est-à-dire comme une réalité propre à la sphère du montrer et non $\mathrm{du}$ démontrer ${ }^{29}$. Or, ce «montrer» fait référence à une attitude éminemment révélatrice, dans le sens où la réalité de la personne n'est pas « démontrée ", mais est 
révélée à travers ses actes concrets. En réalité, l'acte, considéré comme une action consciente dirigée vers un telos spécifique, est précisément ce qui est capable de « réduire la distance entre notre vie et notre vocation ». Cela devient alors une sorte de paradigme d'être une personne :

La personne, telle que je viens de la définir, n'est pas un état, mais un acte. L'homme devient personne dans la mesure où il se manifeste concrètement, d'une façon qui lui est particulière, dans une tâche qui lui est propre et pour laquelle il est responsable ${ }^{30}$.

La personne authentique agit pour atteindre un but et c'est là que réside sa liberté essentielle. Dans la motivation qui anime l'action, le but recherché devient une cause motrice. L'intention est une tension entre la volonté et l'action. Et l'acte présent devient ainsi un témoignage de la convergence entre l'«avenir» at-tendu et le " passé » en-tendu. "Notre vocation est toujours hic et nunc " l'éternité présente ${ }^{32}$.

Dans L'aventure occidentale de l'homme (1957), Rougemont explore plus en détail la question, en se concentrant sur la double genèse (gréco-romaine et chrétienne) de la notion de personne. Avec l'hellénisme, la conception de l'individu en tant qu'être distinct et de la permanence de cet être à travers ses diverses modalités (essence, substance, hypostase) s'était développée. D'autre part, dans le monde romain, le terme "personne ", utilisé en principe pour désigner le masque de l'acteur puis son rôle, en était venu à désigner l'homme lui-même en tant que citoyen. Enfin, les Pères des grands conciles œcuméniques ont effectué la transmutation d'un terme latin ("personne» en tant que valence, définie par son rapport à un contexte) et d'un contenu grec (« individu » en tant qu'atome, existant en soi) dans un dogme exprimant la triple nature et une de la divinité révélée en Jésus, homme et Dieu à la fois :

Les relations que le christianisme définit entre l'homme et «son» Dieu sont personnelles. Dieu est personnel. La Trinité est composée de trois Personnes. Le modèle de toute personne humaine est donné par l'Incarnation du Christ fils de Dieu, en Jésus fils de Marie - Jésus Christ étant à la fois « vrai Dieu et vrai homme $»^{33}$.

24 La personne est ainsi devenue «le fait spécifique et capital de l'anthropologie occidentale $»^{34}$. En fait, suivant le modèle théologique, les premiers philosophes chrétiens ont utilisé le terme pour :

[...] désigner la réalité de l'homme dans un monde christianisé. Car cet homme est, lui aussi, à la fois autonome et en relation. [...] C'est le même Dieu qui, par la vocation qu'il envoie à l'homme, distingue cet homme de tous les autres et le remet en relations concrètes avec ses semblables. [...] Ce qui libère un homme est aussi ce qui le rend responsable vis-à-vis d'autrui. En retour ce qui unit la communauté est aussi ce qui l'oblige à respecter les vocations individuelles.

Nous avons retrouvé, dans cette doctrine de l'homme, les mêmes structures que dans la doctrine de l'Église universelle esquissée plus haut; la même position centrale définissant à la fois l'union et la diversité, l'engagement et la liberté, les droits du tout et les droits des parties ${ }^{35}$.

Nous sommes ici confrontés à une doctrine dialectique et paradoxale de l'existence humaine, qui est donc configurée comme la position centrale à trouver nécessairement afin de limiter les deux dangers « extrêmes » du matérialisme et du spiritualisme, et de leurs déclinaisons politiques actuelles - collectivisme et individualisme. Même le contexte socioculturel du milieu du $\mathrm{XX}^{\mathrm{e}}$ siècle présenterait certaines analogies avec celui de la naissance du christianisme : 
La Grèce individualiste triompha sur les barbares, mais après elle plongea dans l'anarchie. Rome triompha sur l'anarchie et s'écroula sous le poids de son système collectiviste $^{36}$. unique de l'Incarnation, qui porte à la question de l'époque la réponse éternelle de l'Église». Et cette situation «rappelle étrangement la lutte entre démocraties individualistes et États totalitaires $\aleph^{37}$. Par conséquent, il est constamment proposé (dissimulé par les courants ésotériques, par les mouvements philosophiques, par les idéologies politiques) «un double péril simultané: celui de la fuite vers la salut individuel, et celui de l'abandon au sacré collectif, - maladie "grecque" et maladie "romaine" de la personne $\aleph^{38}$. Des maladies auxquelles on ne peut trouver un remède qu'en s'efforçant de « circonscrire le point central, le foyer rayonnant, le cœur de la réalité humaine où vient retentir l'appel des fins les plus lointaines. Et c'est l'acte à la fois immédiat et transcendant de la personne $»^{39}$.

\section{Pour une politique de la personne}

Selon Rougemont, la traduction socio-politique correcte de la philosophie de la personne est le fédéralisme. Son pays d'origine, la Suisse, "cœur battant de l'Europe $\aleph^{40}$, le rend naturellement enclin à adopter l'idée fédérale. Ainsi, lorsqu'il arrive à Paris et entre en contact avec les groupes personnalistes, il devient immédiatement l'un des théoriciens de cette révolution nécessaire que Robert Aron et Arnaud Dandieu ont cherché à traduire en propositions concrètes de réforme sociale ${ }^{41}$. L'hypothèse de base de ce "nouvel ordre ", capable de faire face au " désordre établi ", est impérative et naturelle dans sa formulation: "Le but de la société, c'est la personne $»^{42}$. Alors que l'idéal (souvent inavoué) des sociétés collectivistes serait exactement le contraire : le but de l'homme est la société, l'individu trouve sa raison d'être dans la société.

Cela dit, il est manifeste que « des lois fondées sur la personne sont obligées de tenir compte en premier lieu des diversités personnelles, puis locales, puis régionales... ${ }^{43}$, et donc de se structurer selon une architecture fédérale :

C'est en fait dans le groupe local que la personne peut se réaliser; parce que les tâches civiques se produisent ici à l'échelle de l'individu et qu'un service concret dans la communauté est rendu possible ${ }^{44}$.

À partir de l'expérience de son exil en Amérique pendant la guerre, Rougemont développe la conviction d'une culture européenne spécifique («À ceux qui seraient tentés de nier, a priori, l'originalité de notre culture [...] je répondrai d'un seul mot: Voyagez! Quittez l'Europe, vous la découvrirez! [...] Vue du dehors, l'Europe est évidente $»^{45}$ ) et se rend compte en même temps que, pour préserver cette spécificité, il est nécessaire de lui donner une forme politique communautaire, c'est-à-dire selon une structure fédérale. Selon lui, l'Europe elle-même est la "patrie de la diversité, d'antinomies inséparables: autorité et liberté, personne et communauté, tradition et innovation, droite et gauche, nord et midi, évangélisme et ritualisme, réformisme et révolution, mythe et science, hérésie créatrice et saine doctrine, besoin de sécurité et goût du risque, conformité qui maintient les valeurs et originalité qui les conteste et les rénove $»^{46}$. Elle ne peut donc trouver «son unité paradoxale que dans le libre jeu de ses diversités $»^{47}$, ce qui, traduit en termes politiques, signifie s'organiser selon un système

Études de lettres, $311 \mid 2019$ 
fédéral qui unit les communautés uniquement dans la mesure où leur union peut préserver leur autonomie ${ }^{48}$. L'idée de la tension féconde entre les pôles opposés qui, d'après Rougemont, caractérise la personne se retrouve donc visiblement dans sa conception du fédéralisme.

Persuadé de l'importance de promouvoir une telle cause pour sauvegarder la spécificité culturelle européenne et la préserver d'éventuels conflits futurs, Rougemont y consacrera une grande partie de sa vie et de son engagement intellectuel à partir des années cinquante jusqu'à sa mort. Dès 1948, lors du congrès historique de La Haye présidé par Winston Churchill, il est le rapporteur de la commission de la culture et la résolution qu'il rédige est approuvée à l'unanimité. En 1950, il donne vie au Centre européen de la culture (CEC) dont il sera président à vie. L'année suivante, il fonde l'Association européenne des festivals de musique (AEFM) et participe activement aux travaux préparatoires de ce qui deviendra plus tard le CERN. En 1952, on le voit encore sur une photo, assis entre De Gasperi et Arnold Toynbee, en tant que président de la première table ronde du Conseil de l'Europe ${ }^{49}$.

31 L'engagement concret en faveur de l'Union européenne assurera que les œuvres rougemontiennes des années soixante et soixante-dix soient davantage orientées vers la praxis, et souvent vers des problèmes actuels. Cela les rend paradoxalement plus dépassées que les autres, de nature plus théorique. Cependant, même dans ces écrits, il existe des idées intéressantes et actuelles, comme par exemple dans L'avenir est notre affaire (1977), où le problème de l'écologie est largement traité dans une perspective personnaliste et fédéraliste. Cet intérêt sera davantage cultivé par Rougemont, à tel point qu'il fondera lui-même, avec Jacques Ellul, la première association écologique européenne, ECOROPA.

Cependant, il faut reconnaître que la vision sociopolitique rougemontienne sera destinée à ne pas être mise en œuvre, car ce seront des intérêts de nature tout à fait différente du souci de l'avenir de la culture occidentale et de la sauvegarde de la personne qui l'emporteront au sein du mouvement de fondation de l'Union européenne. La priorité sera accordée à l'intérêt économique et fiscal, selon une mentalité que Rougemont n'aurait pas hésité à condamner comme un retour de doctrines d'abord bourgeoises, puis marxistes. Aujourd'hui, après plus d'un demi-siècle, on peut dire que sa vision ne manquait pas de prophétisme: l'Union européenne, malgré ses mérites pratiques indéniables, présente en effet d'importantes limites culturelles et politiques. L'Europe est perçue comme une institution lointaine, obscure et pas très démocratique, gouvernée par des personnages inconnus de la plupart des citoyens, et pourtant elle prétend légiférer pour les différents États membres, au nom d'une autorité économique présentée comme inattaquable et absolument supérieure à toute question éventuelle de nature sociale, éthique ou juridique. Les chevauchements continus entre la constitution européenne et les constitutions nationales, ainsi que le récent Brexit, en sont la preuve.

Par ailleurs, la dénaturation de la vision fédéraliste européenne a probablement nui d'une part à l'efficacité du mouvement européen tout court, mais de l'autre a contribué à disqualifier la conception même du fédéralisme politique. Rougemont à cet égard avait été le plus prophétique :

Le malheur congénital du fédéralisme reste d'être un concept dialectique, ambigu, et qui autorise - ou incite en tout cas - aux plus invraisemblables pataquès conceptuels ${ }^{50}$. 
Ainsi :

Nous aurons des fédéralistes qui ne penseront qu'à faire l'union et à la renforcer, et nous aurons des fédéralistes qui se soucieront avant tout de protéger les droits de chaque nation ou région contre l'ingérence du pouvoir central. Une nouvelle gauche et une nouvelle droite. Et il faudra constamment rappeler aux deux parties que le véritable fédéralisme ne se trouve dans aucune de ces deux tendances, mais dans leur coexistence acceptée, dans leur dialogue, dans leur tension féconde ${ }^{51}$. pensée politique rougemontienne est réaffirmée, comme nous l'avons vu de toute sa
philosophie de la personne. Ce n'est pas un hasard si, lorsqu'il meurt, il lui restait, encore douze livres à terminer.

En conclusion, Denis de Rougemont a personnellement mené l'aventure risquée d'une pensée qui cherche continuellement à discipliner le conflit en le transformant en harmonie. Il se veut réaliste et à la fois futuriste, pessimiste et pourtant actif ${ }^{52}$, chrétien dans ses racines et "laïque» dans ses fruits, toujours en tension entre le désenchantement barthien pour la misère de l'homme et la conscience pascalienne de sa grandeur inégalée, persuadé au fond que chaque être humain est porteur d'un appel spécifique à accomplir en tant que personne authentique et irremplaçable qui «se dépasse infiniment » dans l'acte même avec lequel elle se révèle à elle-même.

\section{BIBLIOGRAPHIE}

BATAILLE, Georges, La congiura sacra, Torino, Bollati Boringhieri, 2008.

BомBACI, Nunzio, Una vita, una testimonianza. Emmanuel Mounier, Messina, Armando Siciliano, 1999.

BONDI, Damiano, « La Croce e il Kairós. Radici teologiche dell'impegno politico in Denis de Rougemont (1906-1985) », Davar, 5 (2010), p. 115-129.

-, « Persone divine, persona umana. Appunti e spunti per un personalismo trinitario » (<http:// mondodomani.org/teologia/bondi2011.htm>).

DANDIEU, Arnaud, ARON, Roger, La révolution nécessaire, Paris, Grasset, 1933.

LAURENTI, Carlo, « Ré et rétour. Viaggio in estremo occidente ", in Denis de Rougemont, Diario di un intellettuale disoccupato, Roma, Fazi Editore, 1997.

LoCATElli, Silvio, florentis, G. Huen de, Denis de Rougemont. La vita e il pensiero, Milano, Ferro, 1965. LOUBET DEL BAYLE, Jean-Louis, Les non-conformistes des années 30. Une tentative de renouvellement de la pensée politique française, Paris, Éditions du Seuil, 1969.

MOUNIER, Emmanuel, Manifeste au service du personnalisme, Paris, Éditions Montaigne, 1936

(<http://classiques.uqac.ca/>).

MINGARDI, Alberto, Introduction à l'édition italienne des Méfaits de l'instruction publique : I misfatti dell'istruzione pubblica, Rubbettino, Soveria Mannelli (CZ), 2005.

Études de lettres, 311 | 2019 
MORIGI, Silvio, « La “ vertigine della profondità ". “ Interiorizzazione " ed " esteriorizzazione " come " sistole e diastole" della persona in Emmanuel Mounier", in S. S. Macchietti (a cura di), Alla «scula» del personalismo nel centenario della nascita di Emmanuel Mounier, Bulzoni, Roma, 2006. PASSERINI, Luisa, Storie d'amore e d'Europa, Napoli/Roma, L'ancora del Mediterraneo, 2008. RICOEUR, Paul, « Meurt le personnalisme, revient la personne », Esprit, 1 (1983), p. 113-119. ROUGEMONT, Denis de, Les méfaits de l'instruction publique, Eureka, Lausanne, 1972 (1929).

_, Le paysan du Danube, Lausanne, L'Âge d'Homme, 1995 (1932).

-, « Définition de la personne », Esprit, 12 (1934), p. 369-382.

-, Politique de la personne, Paris, Editions Je Sers, 1934.

-, Penser avec les mains, Paris, Albin Michel, 1936.

-, «L'amour action, ou de la fidélité », Esprit, 11 (1938), p. 231-256.

_, « La passion contre le mariage », Esprit, 9 (1938), p. 652-670.

-, L'amour et l'Occident, Paris, Plon, 1939, 1956, 1972.

-, La part du diable, New York, Brentano's, 1942.

-, « Fédéralisme et œcuménisme », Foi et Vie (septembre-octobre 1945), p. 621-639.

-, Les personnes du drame, Neuchâtel, La Baconnière, 1945.

-, L'aventure occidentale de l'homme, Lausanne, L'Âge d'Homme, 2002 (1957).

-, « Originalité de la culture européenne comparée aux autres cultures », Schweizer Monatshefte: Zeitschrift für Politik, Wirtschaft, Kultur, 50 (1960-1961), p. 506-516.

-, The Christian Opportunity, New York, Holt, Rinehart \& Winston, 1963.s

-, Les mythes de l'amour, Paris, Gallimard, 1972 (1967).

-, L'un et le divers ou la cité européenne: deux discours, Neuchâtel, in Écrits sur l'Europe, 2 vols, Paris, Éditions de la Différence, 1994, p. 233-250.

-, «Contribution à une recherche éventuelle sur les sources de la notion d'engagement de l'écrivain », Cadmos (1978), p. 17-25.

-, Inédits, Neuchâtel, La Baconnière, 1988.

-, Denis de Rougemont tel qu'en lui même, in CEuvres complètes de Denis de Rougemont, Écrits sur l'Europe, t. III, vol. II, Paris, Éditions de la Différence, 1994

ROUGEMONT, Denis de, MURET, Charlotte, The Heart of Europe, Duell, Sloan \& Pearce, New York, 1941, p. 826-837.

SPAEMANN, Robert, Personen. Versuche über den Unterschied zwischen "etwas " und " jemand", Stuttgart, J. G. Cotta'sche Buchhandlung Nachfolger, 1996.

\section{NOTES}

1. Politique de la personne, p. 19 sq.

2. Cette subdivision a principalement une valeur fonctionnelle pour le développement ultérieur de notre discussion sur la pensée de Rougemont, celle-ci échappant naturellement à une catégorisation aussi schématique. En réalité, les trois noyaux thématiques identifiés ci-dessus se 
retrouvent souvent ensemble dans presque tous les œuvres du philosophe; il est cependant vrai qu'au cours de l'évolution de sa pensée, il est possible d'identifier certaines phases de développement spécifiques dans lesquelles un noyau thématique acquiert une prépondérance sur les autres.

3. A. Mingardi, Introduction à l'édition italienne des Méfaits de l'instruction publique, p. 19.

4. L'expression Des jeunes gens en colère s'applique généralement aux intellectuels dits «non conformistes des années trente ", dont E. Mounier, A. Marc, A. Dandieu et le même Rougemont. Cf. à ce sujet J.-L. Loubet del Bayle, Les non-conformistes des années 30 ; N. Bombaci, Una vita, una testimonianza, chap. 7 (« Giovani in rivolta »), p. 85-98.

5. "Suite des méfaits », in Les méfaits de l'instruction publique, p. 24.

6. Les méfaits de l'instruction publique.

7. Definition de la personne, p. 373.

8. Ibid., p. 375.

9. E. Mounier, Manifeste au service du personnalisme, p. 41. Nous pouvons donc dire, avec Silvio Morigi, que chez Mounier, « la personne est une énergie formatrice qui est exercée sur la matière de l'individu, sans le supprimer, puisqu'elle s'incarne en lui » (S. Morigi, La «vertigine della profondità », p. 91).

10. Inédits, p. 163.

11. Les mythes de l'amour, p. 273.

12. Les personnes du drame, p. 213.

13. Cf. R. Spaemann, Personen.

14. L'amour et l'Occident, p. 237.

15. Penser avec les mains, p. 103.

16. Politique de la personne, p. 50.

17. Rougemont a fréquenté le Collège de novembre 1937 à juillet 1939 et y a également tenu des conférences. C'est surtout la première partie de L'amour et l'occident qui est influencée par ces rencontres, puisque les chapitres de la seconde partie ont été écrits en premier et qu'ils sont sortis dans une forme presque définitive dans Esprit, numéros de septembre et novembre 1938. Cf. L. Passerini, Storie d'amore e d'Europa, p. 185 ; G. Bataille, La congiura sacra, p. 255 n., 272 n., 281 n., 287 n. ; D. de Rougemont, «La passion contre le mariage » et «L'amour action, ou de la fidélité ».

18. Entretien avec E. Hélisse paru en 1939 dans l'hebdomadaire Tribune de France, cité par L. Passerini, Storie d'amore e d'Europa, p. 185.

19. En 1983, de Rougemont établit une liste des traductions de L'amour et l'Occident, qui réunit des éditions en anglais, néerlandais, italien, espagnol, suédois, allemand, portugais, polonais, serbocroate, roumain et hongrois. Une autre liste non datée rassemble également des éditions en afrikaans, catalan, danois, grec, norvégien, japonais, tchèque, arabe et coréen (cf. L. Passerini, Storie d'amore e d'Europa, p. 216, 373 n., 219). Sur l'influence culturelle du travail rougemontien (attestée par des centaines de lettres et des dizaines de critiques, interviews, films inspirés, etc.), voir Ibid., p. 206-227.

20. L'amour et l'Occident, p. 260.

21. Ibid., p. 39.

22. Ibid., p. 31.

23. Ibid., p. 269.

24. Politique de la personne, p. 181. Pour une compréhension plus profonde de la conception particulière de la dialectique existentielle chez Denis de Rougemont, cf. D. Bondi, « La Croce e il Kairós », p. 203-214.

25. Les personnes du drame, p. 51-104.

26. Le paysan du Danube, p. 16.

27. L'un et le divers ou la cité européenne, in Écrits sur l'Europe, p. 244, vol. II. 
28. Définition de la personne, p. 376. En ce sens, les prophètes, selon Rougemont, sont ceux qui, plus que tout autre homme, réduisent la distance qui sépare la vie de la vocation au point que leur vocation devient toute leur vie, et qu'ils meurent pour l'avoir accomplie. En citant Karl Barth (son père spirituel avec Kierkegaard), selon qui « un prophète se lève et tombe avec sa mission » (er steht und fällt mit seiner Mission), Rougemont affirme que : "Rien ne serait plus ridicule que de tenter de faire la psychologie d'un prophète, ou bien alors elle se réduirait à la syntaxe et au style de son message » (Les personnes du drame, p. 74 n.). Enfin, si « la vocation prophétique des hommes de Dieu qui se lèvent sous l'Ancienne Alliance se confond avec la parole qui les conduira au martyre ", puisque la Parole est leur chemin, leur vérité et leur vie dans ce monde et qu'ils meurent pour l'avoir prononcée et qu'ils n'ont pas d'autre tâche (Ibid., p. 74), cela vaudra donc beaucoup plus pour Celui qui lui-même est la Parole et qui proclame la Nouvelle Alliance avec l'incarnation: «La psychologie de la personne parfaite [Jésus-Christ] se réduirait purement et simplement à son histoire, à l'énoncé des témoignages visibles qu'elle produit [les Évangiles] » (Définition de la personne, p. 375).

29. Cf. P. Ricoeur, « Meurt le personnalisme, revient la personne ».

30. Politique de la personne, p. $180 \mathrm{sq}$.

31. L'amour et l'Occident, p. 378. Hic et Nunc était le titre de la revue d'inspiration barthienne fondée par Rougemont en 1932, avec Henry Corbin et Roland de Pury entre autres.

32. Les personnes du drame, p. 66.

33. Les mythes de l'amour, p. 14.

34. L'aventure occidentale de l'homme, p. 61. Pour approfondir la "genèse théologique " de la personne et ses implications théologiques et philosophiques, voir D. Bondi, «Persone divine, persona umana ».

35. « Fédéralisme et œcuménisme », p. 630.

36. « Fédéralisme et œucuménisme », p. 630.

37. The Christian Opportunity, traduit de l'italien.

38. L'aventure occidentale de l'homme, p. 64.

39. Penser avec les mains, p. 249.

40. Cf. D. de Rougemont, Ch. Muret, The Heart of Europe.

41. Cf. A. Dandieu, R. Aron, La révolution nécessaire.

42. Politique de la personne, p. 23.

43. Ibid., p. 58.

44. The Christian Opportunity, traduit de l'italien.

45. «Originalité de la culture européenne », p. 507.

46. L'un et le divers, p. 245.

47. «Originalité de la culture européenne », p. 508.

48. L'amour et l'Occident, p. 463.

49. Cf. C. Laurenti, Ré et rétour.

50. L'un et le divers, p. 238.

51. L'avvenire del federalismo, p. 78, traduit de l'italien.

52. Rougemont lui-même aimait se définir, in Politique de la Personne, comme un "pessimiste actif »; et encore en septembre 1985, peu avant sa mort, dans la dernière interview accordée à Guido Ferrari, il précisera : «Je reste pessimiste actif, mai je souligne peut-être plus le mot " actif" que le mot " pessimiste" " (Denis de Rougemont tel qu'en lui même, in Écrits sur l'Europe, p. 836, vol. II). Au contraire, Mounier définissait son attitude philosophique comme un « optimisme tragique ». 


\section{RÉSUMÉS}

Cet article vise à examiner l'ensemble de la pensée de Rougemont selon trois perspectives principales : tout d'abord, la naissance «négative» du personnalisme en tant que réaction politique à la fois au totalitarisme et à l'individualisme; deuxièmement, la recherche d'une proposition philosophique positive en tant que soutien théorique à l'exercice d'actions sociales et culturelles ; troisièmement, l'engagement politique personnel de Rougemont dans le processus d'unification européenne. En fin de compte, nous soulignons pourquoi et comment la connaissance et l'étude de cet auteur peuvent être non seulement enrichissantes sur le plan personnel, mais également très importantes pour mieux comprendre la situation actuelle de l'Union européenne et les possibilités de la modifier. 